www.jusletter.ch

Sebastian Reichle / Bernhard Stehle

\title{
Coronavirus und Geschäftsraummiete
}

In den vergangenen Wochen ist eine Diskussion darüber entbrannt, ob und wie sich die gestützt auf Notrecht angeordneten Betriebsschliessungen zur Eindämmung der Ausbreitung und Übertragung des Coronavirus in der Schweiz auf laufende Geschäftsmietverhältnisse auswirken. Dieser Beitrag geht der Frage nach, ob das Obligationenrecht Mietern (und Pächtern) von Geschäftsräumen (z.B. Ladengeschäfte oder Restaurants) eine Handhabe gibt, auch gegen den Willen des Vermieters eine Senkung oder gar den Erlass des Mietzinses für die Zeit durchzusetzen, während der sie ihre (reguläre) Geschäftstätigkeit aufgrund der behördlich angeordneten Betriebsschliessung nicht ausüben können.

Beitragsart: Beiträge

Rechtsgebiete: Miet- und Pachtrecht, Obligationenrecht

Zitiervorschlag: Sebastian Reichle / Bernhard Stehle, Coronavirus und Geschäftsraummiete, in: Jusletter 18. Mai 2020 


\section{Inhaltsübersicht}

1. Zusammenfassung

2. Ausgangslage

3. Rechtliche Einordnung

3.1. Spezifische mietvertragliche Abmachungen

3.2. Mögliche anwendbare Rechtsfiguren im schweizerischen Obligationenrecht

3.3. Möglichkeit 1: Anwendung des Art. 119 OR

3.3.1. BGE 57 II 532: Unvorhersehbarer Wegfall der Berufszulassung

3.3.2. BGE 62 II 42: Behördliches Betriebsverbot

3.3.3. Schlussfolgerung

3.4. Möglichkeit 2: Herabsetzungsanspruch wegen Mangel an der Mietsache

3.4.1. Abgrenzung und Verhältnis zu Art. 119 OR

3.4.2. Begriff des Mangels

3.4.3. Schlussfolgerung

3.5. Möglichkeit 3: Vertragsanpassung, clausula rebus sic stantibus

3.5.1. Abgrenzung und Verhältnis zu weiteren Rechtsinstituten

3.5.2. Voraussetzungen der Anpassung

3.5.3. Ausmass der Anpassung

4. Fazit

\section{Zusammenfassung}

[1] Coronavirus und Leistung des Vermieters

- Der Vermieter muss dem Mieter eine Sache zum Gebrauch überlassen (Art. 253 OR). Er muss ihm die Sache zum vereinbarten Zeitpunkt in einem zum vorausgesetzten Gebrauch tauglichen Zustand übergeben und in demselben Zustand erhalten (Art. 256 Abs. 1 OR).

- Die Mietsache muss also nach ihrer Beschaffenheit, ihrem Zustand und ihrer Lage dem Mieter die vertraglich vorgesehene Nutzung ermöglichen. Für diese objektbezogenen Umstände trägt der Vermieter die Verantwortung.

- Darüber hinaus trägt der Vermieter aber mangels einer besonderen Vereinbarung (z.B. einer entsprechenden Zusicherung oder einer Umsatzmiete) nicht das Risiko, dass der Mieter die Mietsache auch tatsächlich bestimmungsgemäss verwenden kann. Dieses Verwendungsrisiko liegt beim Mieter.

- Ist die Mietsache aufgrund eines objektbezogenen Umstands nur noch eingeschränkt tauglich für die vertragsgemässe Nutzung, so ist sie mangelhaft (Art. 259 ff. OR). Der Mieter kann vom Vermieter unter anderem verlangen, dass dieser den Mietzins herabsetzt. Beispiele für solche Umstände sind Bau- oder Fluglärm (denn sie betreffen die Lage des Mietobjekts).

- Ist die Überlassung der Mietsache unmöglich geworden (z.B. weil das Haus, in dem sich die Mieträumlichkeiten befanden, niederbrannte), so ist der Vermieter von seiner Leistung befreit und entsprechend auch der Mieter seiner Zahlungspflicht enthoben (Art. 119 OR).

- Die Covid-19-bedingten Betriebsschliessungen sind keine objektbezogenen Umstände: Sie betreffen nicht die Beschaffenheit, den Zustand oder die Lage des Mietobjekts. Entsprechend sind Geschäftsräume aufgrund dieser Betriebsschliessungen nicht mangelhaft.

- Sodann verunmöglichen die Betriebsschliessungen die Leistung des Vermieters nicht: Er kann nach wie vor Geschäftsräume zur Verfügung stellen, die für die vertragsgemässe Nut- 
zung geeignet sind. Mangels einer anderslautenden Vereinbarung zwischen Vermieter und Mieter trägt der Mieter das Verwendungsrisiko der Räumlichkeiten.

[2] Coronavirus und Leistung des Mieters

- Der Mieter schuldet dem Vermieter den Mietzins für die Mietsache (Art. 253 OR).

- Diese Leistung wurde aufgrund der Covid-19-bedingten Betriebsschliessungen nicht unmöglich. Geldleistungen können nicht unmöglich werden. Geld hat man zu haben.

- Die Pflicht zur Zahlung des Mietzinses kann jedoch aufgrund der Betriebsschliessung unzumutbar werden.

- In diesen Fällen können die Parteien nicht mehr nach Treu und Glauben davon ausgehen, der Vertrag verteile das Verwendungsrisiko immer noch einseitig zu Lasten des Mieters. Der Vertrag enthält vielmehr eine Lücke.

- Der Mieter kann nun verlangen, dass der Vertrag angepasst bzw. ergänzt und der Mietzins herabgesetzt wird (z.B. auf eine Höhe, welche einer Lagernutzung entspricht).

- Massgebend für diese Möglichkeit der Vertragsanpassung sind die Voraussetzungen der clausula rebus sic stantibus.

\section{Ausgangslage}

[3] Um die Verbreitung des Coronavirus einzudämmen, hat der Bundesrat am 13. März 2020 gestützt auf Art. 185 Abs. 3 Bundesverfassung (BV) und Art. 7 Epidemiengesetz (EpG) ${ }^{1}$ unter anderem Verbote von Veranstaltungen mit 100 oder mehr Teilnehmenden erlassen. ${ }^{2}$ Schon nach wenigen Tagen erwiesen sich diese ersten Massnahmen infolge der rasch um sich greifenden Seuche als ungenügend. Der Bundesrat sah sich daher gezwungen, die «ausserordentliche Lage» im Sinne des Epidemiengesetzes auszurufen und mit Wirkung ab dem 17. März 2020 erheblich weiter gehende Massnahmen zu verhängen. Unter anderem verbot er Präsenzveranstaltungen in Schulen, Hochschulen und übrigen Ausbildungsstätten sowie private und öffentliche Veranstaltungen in genereller Weise und ordnete die Schliessung öffentlich zugänglicher Einrichtungen für das Publikum an. Um die Grundversorgung aufrechtzuerhalten, durften jedoch bestimmte Einrichtungen während des Lockdowns betrieben und einzelne Veranstaltungen noch durchgeführt werden. Namentlich Lebensmittelläden, Take-aways, Apotheken, Banken oder Poststellen konnten ihre Leistungen durchgängig in den bisher genutzten Räumlichkeiten anbieten. Einschränkungen ergaben sich für diese Betriebe einzig durch die Verpflichtung, dafür zu sorgen,

1 Bundesgesetz über die Bekämpfung übertragbarer Krankheiten des Menschen vom 28. September 2012, SR 818.101.

2 Vgl. Medienmitteilung des Bundesrats vom 13. März 2020 (https://www.admin.ch/gov/de/start/dokumentation/ medienmitteilungen/bundesrat.msg-id-78437.html, Website zuletzt besucht am 12. Mai 2020) sowie Art. 6 Abs. 1 der Verordnung 2 vom 13. März 2020 über Massnahmen zur Bekämpfung des Coronavirus (COVID-19-Verordnung 2; SR 818.101.24). 
dass die Empfehlungen des Bundesamtes für Gesundheit hinsichtlich Hygiene und sozialer Distanz eingehalten werden. ${ }^{3}$

[4] Diese Massnahmen, die die Freiheitsrechte der Bürger erheblich einschränkten, haben ihre Wirkung nicht verfehlt. Die Verbreitung des Coronavirus verlangsamte sich infolgedessen stark. Angesichts dieser Entwicklung entschied der Bundesrat in seinen Sitzungen vom 8. und 16. April 2020, die angeordneten Massnahmen schrittweise wieder zu lockern. ${ }^{4}$ Bereits ab dem 27. April 2020 durften insbesondere Baumärkte und Coiffeursalons ihre Leistungen unter Einhaltung branchenspezifischer Schutzkonzepte wieder anbieten. Ab dem 11. Mai 2020 dürfen sämtliche Läden wieder Kunden vor Ort bedienen. Ab diesem Datum dürfen auch Restaurants wieder Gäste empfangen (freilich nur unter Einhaltung gewisser Vorgaben: maximal vier Personen pro Tisch oder Eltern mit Kindern und zwei Meter Abstand zwischen den Tischen). Der Betrieb einer Bar oder eines Kinos ist dagegen aktuell immer noch verboten. Der Bundesrat hat in Aussicht gestellt, am 27. Mai 2020 über weitere Öffnungsschritte zu entscheiden. ${ }^{5}$

[5] Zahlreichen Betrieben wie Fahrschulen, Kosmetikstudios, Restaurants, Blumengeschäften sowie etwa Kleiderläden war es somit ab dem 17. März 2020 für eine beschränkte Zeit nicht mehr erlaubt, ihre zentrale Geschäftstätigkeit in den gemieteten Räumlichkeiten anzubieten. Die Erbringung gewisser Leistungen blieb für einzelne Betriebe freilich während des Lockdowns möglich. Einerseits konnten - zumindest theoretisch - bestimmte Leistungen, wie zum Beispiel der Verkehrskundeunterricht einer Fahrschule, auf digitalem Wege erbracht werden. Andererseits blieben für gewisse Betriebe bestimmte Tätigkeiten in den Geschäftsräumlichkeiten noch zulässig. Restaurationsbetriebe durften etwa ihre Leistungen als Take-away oder als Hauslieferdienst anbieten. Aus zeitlichen und betrieblichen Gründen kamen entsprechende Anpassungen der Geschäftstätigkeit jedoch nur in wenigen Fällen in Betracht. Erhebliche Umsatzeinbrüche konnten damit oft kaum verhindert werden.

[6] Unter diesen Umständen gerieten schon bald die Geschäftsmieten in den Vordergrund der Diskussion. Oftmals machen diese einen beträchtlichen Teil der Fixkosten eines Betriebs aus. Infolge der weitreichenden, zahlreiche Unternehmen betreffenden Betriebsverbote entbrannte vor allem eine Diskussion darüber, ob Geschäftsmieter infolge der behördlich angeordneten Betriebsschliessungen eine Senkung oder gar den Erlass des Mietzinses geltend machen können.

[7] Der Mieterverband stellt sich unter anderem auf den Standpunkt, dass von einem Mangel an der Mietsache auszugehen sei, wenn Geschäfte kraft öffentlich-rechtlichen Verbots generell schliessen müssen. Mieter betroffener Geschäfte hätten ein Anrecht auf Herabsetzung oder Erlass des Mietzinses gegenüber dem Vermieter, auch wenn keine der beiden Parteien für diese Situation ein Verschulden treffe. ${ }^{6}$

3 Vgl. Medienmitteilung des Bundesrats vom 16. März 2020 (https://www.admin.ch/gov/de/start/dokumentation/ medienmitteilungen.msg-id-78454.html, Website zuletzt besucht am 12. Mai 2020) sowie Art. 5 und 6 der Verordnung 2 vom 13. März 2020 über Massnahmen zur Bekämpfung des Coronavirus (COVID-19-Verordnung 2; SR 818.101.24) (Stand 17. März 2020).

4 Medienmitteilungen des Bundesrats vom 8. April 2020 (https://www.bag.admin.ch/bag/de/home/das-bag/ aktuell/medienmitteilungen.msg-id-78744.html, Website zuletzt besucht am 20. April 2020) und vom 16. April 2020 (https://www.bag.admin.ch/bag/de/home/das-bag/aktuell/medienmitteilungen.msg-id-78818.html, Website zuletzt besucht am 25. April 2020).

5 Medienmitteilung des Bundesrats vom 29. April 2020 (https://www.bag.admin.ch/bag/de/home/das-bag/aktuell/ medienmitteilungen.msg-id-78948.html, Website zuletzt besucht am 6. Mai 2020).

6 Sarah Brutschin/Xavier Rubli/Pierre Stastny, Bezahlung des Mietzinses für Geschäftsräume während der Covid-19-Epidemie, Rechtsgutachten März 2020 (https://www.mieterverband.ch/mv/mietrecht-beratung 
[8] Nach Ansicht des Hauseigentümerverbandes (HEV) Schweiz führen die notrechtlich angeordneten Betriebsschliessungen dagegen nicht zu einem Mangel an der Mietsache. ${ }^{7}$ Dabei stützt sich der HEV Schweiz auf die bei Prof. Dr. iur. Peter Higi eingeholte gutachterliche Stellungnahme zur Frage der Herabsetzung des Mietzinses wegen Mängeln des Geschäftsraums im Zusammenhang mit der «Corona-Pandemie». Gemäss diesem Gutachten ist ausschlaggebend, dass es nicht am Mietlokal bzw. dessen Zustand liege, wenn ein Geschäftsraummieter wegen behördlicher Anordnungen keine Kunden mehr empfangen könne, sondern an der vom Mieter ausgeübten Geschäftstätigkeit, die zeitweise nicht mehr erlaubt sei. Es fehle insoweit leicht erkennbar an einer Grundlage für die Herabsetzung des Mietzinses wegen Mangelhaftigkeit der Sache. ${ }^{8}$

[9] Hinzuweisen ist darauf, dass der Bundesrat am 27. März 2020 gestützt auf Art. 185 Abs. 3 BV die Verordnung über die Abfederung der Auswirkungen des Coronavirus im Miet- und Pachtwesen erlassen hat. ${ }^{9}$ Gemäss Abs. 2 dieser Verordnung betragen Zahlungsfristen, die Vermieter von Geschäftsräumen für zwischen dem 13. März 2020 und dem 31. Mai 2020 fällig werdende Mietzinsen oder Nebenkosten ansetzen, in Abweichung zu Art. 257d Abs. 1 OR mindestens 90 Tage, insofern die betreffenden Mieter wegen der Massnahmen des Bundesrates zur Bekämpfung des Coronavirus in Zahlungsrückstand geraten sind. Eine vergleichbare Regelung, freilich mit einer Zahlungsfrist von 120 Tagen, gilt gemäss Abs. 4 der erwähnten Verordnung auch für die Bezahlung von Pachtzinsen. Unklar blieb zunächst, ob der Bundesrat noch weiter gehende Regelungen zur Tragung der Mietzinse während den notrechtlich verordneten Betriebsschliessungen erlassen würde. Mit Medienmitteilung vom 8. April 2020 stellte er klar, in diesem Zusammenhang nicht weiter in die privatrechtlichen Beziehungen zwischen den Mietparteien eingreifen zu wollen, und rief diese gleichzeitig dazu auf, im Dialog konstruktive und pragmatische Lösungen zu suchen. ${ }^{10}$

[10] Angesichts der Komplexität und Verschiedenartigkeit der zahlreichen betroffenen Mietverhältnisse ist es zu begrüssen, dass der Bundesrat nicht vorschnell Regelungen erlassen hat, die den verschiedenen Einzelfällen nicht ausreichend Rechnung zu tragen vermögen. In vielen Fällen dürfte es den Mietparteien gelingen, pragmatische und sachgerechte Lösungen zu finden, die für beide Seiten stimmen. Für die Fälle, in denen dies nicht gelingt, ist - vorbehalten eines durch das schweizerische Parlament geregelten Mieterlasses ${ }^{11}$ - die geltende Rechtslage massgebend.

/ratgeber-mietrecht/fallbeispiele/a-d/corona-geschaeftsraummiete-mietzinsreduktion.html, Website zuletzt besucht am 5. April 2020), N $1.1 \mathrm{ff}$.

7 Mediencommuniqué HEV Schweiz vom 30. März 2020, «Betriebsschliessung wegen Corona stellt keinen Mangel an der Mietsache dar» (https://www.hev-schweiz.ch/fileadmin/sektionen/hev-schweiz/PDFs_Dateien/ Medienmitteilungen/2020_Medienmitteilungen/2020_03_30_MM_Betriebsschliessung_wegen_Corona_stellt_ keinen_Mangel_an_der_Mietsache_dar.pdf, Website zuletzt besucht am 5. April 2020); vgl. auch David LACHAT, Avis de droit de Me Marc IYNEDJIAN, Gutachterliche Stellungnahme vom 1. April 2020 [https://www.asloca. ch/wp-content/uploads/2020/04/Commentaire-Lachat-sur-avis-de-droit-Iynedjian-de-la-FRI_compressed.pdf, Website zuletzt besucht am 5. April 2020).

8 Peter Higi, Gutachterliche Stellungnahme zur Frage der Herabsetzung des Mietzinses wegen Mängeln des Geschäftsraums im Zusammenhang mit der «Corona-Pandemie» vom 26. März 2020 (https://www.hev-schweiz.ch/ fileadmin/sektionen/hev-schweiz/PDFs_Dateien/Medienmitteilungen/2020_Medienmitteilungen/Gutachterl_ Stellungn_Prof_Higi.pdf, Website zuletzt besucht am 5. April 2020).

9 Verordnung vom 27. März 2020 über die Abfederung der Auswirkungen des Coronavirus im Miet- und Pachtwesen (COVID-19-Verordnung Miete und Pacht; SR 221.213.4).

10 Medienmitteilung des Bundesrats vom 8. April 2020 (https://www.admin.ch/gov/de/start/dokumentation/ medienmitteilungen.msg-id-78746.html, Website zuletzt besucht am 11. April 2020).

11 Im schweizerischen Parlament ist ein beträchtlicher Teil gewillt, den Erlass von Mieten für zwangsgeschlossene Betriebe gesetzlich zu regeln. Uneinigkeit besteht vor allem darin, wie eine entsprechende Lösung aussehen soll. Vgl. hierzu NZZ vom 5. Mai 2020, «Nationalrat und Ständerat fordern den Erlass von Mieten für leidende Betriebe» 
Hier setzt der vorliegende Beitrag an. Er zeigt auf, welche Gesichtspunkte und Umstände in der juristischen Beurteilung entsprechender Auseinandersetzungen massgebend sein dürften.

\section{Rechtliche Einordnung}

[11] Wird ein Mieter während des laufenden Mietverhältnisses durch gesetzgeberische oder behördliche Massnahmen zur Bekämpfung einer grassierenden Pandemie in seiner Nutzung eingeschränkt, stellt sich die Frage, ob und inwieweit der Vermieter und/oder der Mieter die Folgen dieser Einschränkung zu tragen hat.

\subsection{Spezifische mietvertragliche Abmachungen}

[12] Zunächst ist auf den Mietvertrag abzustellen. Es ist zu untersuchen, ob die Parteien die Folgen staatlich angeordneter Betriebsschliessungen im Zusammenhang mit Pandemien, Epidemien usw. auf das Mietverhältnis individuell geregelt haben. Denkbar wären etwa spezifische Risikotragungsklauseln oder Zusicherungen, wonach die Miete nicht geschuldet ist, wenn von staatlicher Seite im Falle einer Epidemie Betriebsschliessungen angeordnet werden. Es ist naheliegend, dass Mietverträge regelmässig keine solchen Bestimmungen enthalten, zumal sie häufig vom Vermieter aufgesetzt werden. Aber auch Klauseln, die das Pandemierisiko auf den Mieter überwälzen, dürften sehr selten sein. ${ }^{12}$ Erwähnenswert ist in diesem Zusammenhang, dass mit der Vereinbarung einer Umsatzmiete der Vermieter das Risiko von staatlich angeordneten Betriebsschliessungen mitträgt. Denn wenn kein Umsatz erzielt wird, ist folglich auch kein umsatzabhängiger Mietzins geschuldet.

\subsection{Mögliche anwendbare Rechtsfiguren im schweizerischen Obligatio- nenrecht}

[13] Die Parteien dürften also nur in den wenigsten Fällen spezifische vertragliche Vorkehrungen für ausserordentliche Situationen wie die Coronavirus-Pandemie getroffen haben. Es stellt sich daher die Frage, wie solche Sachverhalte gemäss geltendem Obligationenrecht zu erfassen sind.

[14] Drei Rechtsfiguren spielen hinsichtlich der Frage des Erlasses oder der Senkung des Mietzinses potenziell eine Rolle und sind näher zu prüfen:

- die unverschuldete nachträgliche (temporäre) Unmöglichkeit gemäss Art. 119 OR;

- der mietrechtliche Mangel gemäss Art. 258 ff. OR und gestützt darauf der Herabsetzungsanspruch gemäss Art. 259d OR;

(https://www.nzz.ch/wirtschaft/corona-krise-parlament-fordert-mietzinserlasse-fuer-das-gewerbe-ld.1555033, Website zuletzt besucht am 6. Mai 2020).

12 Falls Betriebsschliessungen aufgrund von Pandemien als Mängel qualifiziert werden (dazu Titel 3.4), dürften solche Klauseln aufgrund von Art. 256 Abs. 2 OR und (den relativ zwingenden) Art. 258 ff. OR unzulässig sein, s. Peter Higi/Anton Bühlmann, in: Zürcher Kommentar zum Obligationenrecht, Die Miete, Vorbemerkungen zum 8. Titel (Art. 253-273c OR), Art. 253-265 OR, 5. Aufl., Zürich 2019 (zit. ZK OR-Verfasser), Art. 256 N 58 und Art. 259a N 6. 
- die aus Art. 2 Abs. 1 ZGB (Handeln nach Treu und Glauben) abgeleitete «clausula rebus sic stantibus».

[15] Zu beurteilen ist somit, ob durch die vom Bundesrat verhängten Betriebsschliessungen

- die Leistung des Vermieters temporär unmöglich geworden ist und daher auch kein oder nur noch ein verminderter Mietzins geschuldet ist (Möglichkeit 1);

- ein Mangel an der Mietsache besteht, der zum Erlass oder zur Herabsetzung des Mietzinses berechtigt (Möglichkeit 2);

- oder sich die Umstände in unvorhersehbarer Weise derart geändert haben, dass infolgedessen der Mietzins teilweise oder gar nicht mehr geschuldet ist (Möglichkeit 3).

\subsection{Möglichkeit 1: Anwendung des Art. 119 OR}

[16] Art. 119 Abs. 1 OR schreibt vor, dass die Leistung des Schuldners als erloschen gilt, wenn sie durch Umstände, die der Schuldner nicht zu vertreten hat, unmöglich geworden ist. Nach Abs. 2 der Bestimmung verliert der Schuldner im Gegenzug auch seine Forderung und muss bereits empfangene Leistungen zurückerstatten. Massgebend ist also die Frage, ob die Leistung des Schuldners unmöglich wird.

[17] Die Leistung des Mieters (Zahlung des Mietzinses) kann nicht unmöglich werden, denn es gilt bekanntlich der Grundsatz, dass man Geld haben muss. ${ }^{13}$ Demgegenüber kann in laufenden Miet- oder Pachtverhältnissen die Leistung des Vermieters oder Verpächters unmöglich werden, z.B. infolge vollständigen Niederbrennens der gemieteten Büroräumlichkeiten. Falls keine der Parteien diese Unmöglichkeit zu vertreten hat, gilt die Schuld des Vermieters, dem Mieter die entsprechenden Räumlichkeiten zum Gebrauch zu überlassen, als erloschen (Art. 119 Abs. 1 OR). Der Mieter schuldet im Gegenzug gemäss Art. 119 Abs. 2 OR keine Mietzinsen mehr. ${ }^{14}$

[18] Zu prüfen ist nun, ob die COVID-19-Verordnung 2 eine Unmöglichkeit im Sinne von Art. 119 OR verursacht hat.

[19] Vorab ist darauf hinzuweisen, dass es umstritten ist, ob auch eine vorübergehende Unmöglichkeit unter Art. 119 OR subsumiert werden kann. ${ }^{15}$ Gemäss bundesgerichtlicher Rechtsprechung kommt Unmöglichkeit nicht in Betracht, wenn ihr Wegfall absehbar ist:

«An sich ist denkbar, dass auch diese Duldungspflicht unmöglich werden kann [...]. Dies setzt indessen voraus, dass die Unmöglichkeit entweder mit Gewissheit bis zum Vertragsende bestehen bleibt oder ihr Wegfall zumindest nicht abzusehen ist [... ]. Gemäss $§ 65$ Abs. 1 $P B G / Z H$ soll in der Reservezone eine bestimmte Nutzung erst später zugelassen werden. Die Grundeigentümer haben periodisch - frühestens nach acht Jahren seit der Zuweisung - einen gesetzlichen Anspruch auf Überprüfung des Bauverbotes (§ 65 Abs. 4 PBG/ZH).

13 Urteil des Bundesgerichts 4C.344/2002 vom 12. November 2003 E. 4.2; Brutschin/Rubli/Stastny (Fn. 6), N 2.3.

14 Ausführlich hierzu Andrea Haefeli/Dario Galli/Markus Vischer, Coronavirus SARS-CoV-2: Klärung mietrechtlicher Fragen, in: Jusletter 14. April 2020, N 13.

15 Vgl. Brutschin/Rubli/Stastny (Fn. 6), N 2.6. 
Bezogen auf die hundertjährige Vertragsdauer ist die Dauer des Bauverbotes daher nicht unabsehbar. Eine Unmöglichkeit im Sinne von Art. 119 Abs. 1 OR ist deshalb zu verneinen.» 16

[20] Der Bundesrat hat im Zusammenhang mit dem Lockdown bereits erste Lockerungsschritte in die Wege geleitet. Weitere sind absehbar. Art. 119 OR ist dementsprechend gemäss bundesgerichtlicher Rechtsprechung wohl gar nicht anwendbar.

[21] Davon abgesehen ist die Unterscheidung zwischen Zweckverfehlung und Verwendungsunmöglichkeit entscheidend. Zweckverfehlung liegt vor, wenn der im Rahmen eines Schuldverhältnisses zu erzielende Leistungserfolg, der zum Inhalt der geschuldeten Leistung gehört, nicht mehr eintreten kann. Blosse Verwendungsunmöglichkeit ist dagegen anzunehmen, wenn der Schuldner die Sache nicht für den vorgesehenen Zweck gebrauchen kann, dieser Zweckgebrauch jedoch nicht Inhalt der geschuldeten Leistung ist. ${ }^{17}$ Während Zweckverfehlung als ein Fall der absoluten Unmöglichkeit zu betrachten ist (da damit die Leistung des Schuldners unmöglich wird), liegt im Fall der blossen Verwendungsunmöglichkeit keine Unmöglichkeit im Sinne von Art. 119 OR vor (da die Leistung des Schuldners möglich bleibt). Der Mieterverband argumentiert unter anderem mit dem Begriff der Verwendungsunmöglichkeit, ${ }^{18}$ bezieht sich jedoch nach den hier verwendeten Definitionen vielmehr auf die Rechtsfigur der Zweckverfehlung.

[22] Müssen Mieter aufgrund der notrechtlich angeordneten Betriebsschliessungen ihre Geschäftstätigkeit vorübergehend einstellen, so ist mit Blick auf die vorangegangenen Ausführungen die entscheidende Frage, ob die tatsächliche Verwendung der Räumlichkeiten für einen bestimmten Zweck zum Inhalt der vom Vermieter geschuldeten Leistung gehört oder nicht. Kommt man zum Schluss, dass der Vermieter dem Mieter nicht nur zweckgeeignete Räumlichkeiten überlassen muss, sondern zudem dafür verantwortlich ist, dass der Mieter diese auch tatsächlich entsprechend verwenden kann, käme Zweckverfehlung in Betracht. Da diesfalls von gänzlicher oder teilweiser Unmöglichkeit auszugehen wäre, müsste der Mietzins entsprechend ganz oder teilweise wegfallen. Gehört demgegenüber die tatsächliche Verwendungsmöglichkeit nicht zur Leistungspflicht des Vermieters, so wird dessen Leistung auch nicht unmöglich und die Befreiungswirkungen von Art. 119 OR treten nicht ein. Zu Recht weist Koller darauf hin, dass es bei Zweckverfehlung und Verwendungsunmöglichkeit nicht primär um ein Unmöglichkeitsproblem gehe, sondern um ein solches der Leistungsbestimmung und damit um ein Problem der Vertragsauslegung. ${ }^{19}$

[23] In der Zwischenkriegszeit beurteilte das Bundesgericht zwei Fälle nach den dargelegten Wertungsgesichtspunkten. In beiden Fällen ging es um die mietrechtlichen Auswirkungen öffentlichrechtlicher Bestimmungen, die während laufenden Mietverhältnissen in Kraft traten und den Mietern die Ausübung ihres Geschäfts in den gemieteten Räumlichkeiten verunmöglichten. Das Bundesgericht kam in diesen beiden Fällen jedoch nicht zum selben Ergebnis. Deshalb sind sie für die vorliegende Frage besonders aufschlussreich.

16 Urteil des Bundesgerichts 4C.34/2000 vom 24. April 2001 E. 4 (in BGE 127 III 300 nicht publ. Erw.).

17 Alfred Koller, Schweizerisches Obligationenrecht Allgemeiner Teil, Handbuch des allgemeinen Schuldrechts, 4. Aufl., Bern 2017, N 53.14.

18 Brutschin/Rubli/Stastny (Fn. 6), N 2.4.

19 Koller (Fn. 17), N 53.15. 


\subsubsection{BGE 57 II 532: Unvorhersehbarer Wegfall der Berufszulassung}

[24] Ein Zahnarzt als Vermieter und eine Inhaberin des zürcherischen Zahnarztpatents als Mieterin schlossen am 5. Oktober 1925 einen Mietvertrag über eine im glarnerischen Mollis gelegene Zahnarztpraxis ab. Der Vertrag wurde für die Dauer von drei Jahren, vom 1. November 1925 bis 1. November 1928, abgeschlossen, mit der Abrede, dass sich die Vertragsdauer mangels rechtzeitiger Kündigung jeweils stillschweigend um ein Jahr verlängere. Die Zahnärztin betrieb in der Folge die zahnärztliche Praxis, welche damals im Kanton Glarus an keine Befähigungsausweise oder sonstigen Voraussetzungen gebunden war. Am 1. Mai 1927 erliess die glarnerische Landsgemeinde ein Gesetz, wonach ab dem 1. Januar 1928 die gewerbsmässige Ausübung der zahnärztlichen Praxis nur noch Personen gestattet wurde, die das eidgenössische Diplom für Zahnärzte erworben hatten. Der Mieterin wäre es zudem aufgrund ihres Geschlechts selbst bei bestandener eidgenössischer Prüfung verwehrt geblieben, weiterhin im Kanton Glarus als Zahnärztin zu praktizieren. Sie teilte dem Vermieter infolgedessen mit, dass sie den Vertrag wegen höherer Gewalt als beendet ansehe und demgemäss zurücktrete. Als Grund führte sie an, der Vertrag habe die Möglichkeit der Berufsausübung als Zahnärztin zur notwendigen Voraussetzung gehabt. ${ }^{20} \mathrm{Ab}$ Inkrafttreten des neuen Gesetzes zahlte sie den Mietzins nicht mehr. Der Vermieter klagte diesen zufolge Vertragsbruchs ein.

[25] Das Bundesgericht führte in seinen Erwägungen aus, «dass die Beklagte das fragliche Heimwesen miete zum Zwecke, darin die bis anhin vom Kläger geführte Zahnarztpraxis weiter zu betreiben. Die Möglichkeit, diesen Beruf daselbst ausüben zu können, war daher eine notwendige Voraussetzung des Vertrages. Hierzu war indessen die Beklagte angesichts des erwähnten von der Landsgemeinde am 1. Mai 1927 genehmigten Gesetzes vom 1. Januar 1928 an nicht mehr in der Lage, da sie nicht Inhaberin des eidg. Zahnarztdiploms ist. Es fragt sich nun, ob die Beklagte diese in ihrer Person gelegene Unmöglichkeit zu vertreten habe oder nicht. Wenn dies verneint werden muss, ist ihr auch nicht zuzumuten, weiterhin den Mietzins für die Liegenschaft, die sie nicht mehr vertragsgemäss benützen kann, zu bezahlen; denn Art. 119 OR schreibt vor, dass eine Forderung als erloschen gelte, sofern durch Umstände, die der Schuldner nicht zu verantworten hat, seine Leistung unmöglich geworden ist. Hierunter ist aber nicht nur eine absolute Unmöglichkeit zu verstehen, es genügt die relative, wonach die Leistung mit den einem Schuldner zuzumutenden Opfern nicht bewirkt werden kann, da ein Gläubiger vom Schuldner nicht mehr verlangen kann, als die bona fides gebietet». ${ }^{21}$ Gestützt auf diese Begründung wies das Bundesgericht die Klage ab und schützte damit die von der Mieterin geltend gemachte vorzeitige Vertragsbeendigung.

\subsubsection{BGE 62 II 42: Behördliches Betriebsverbot}

[26] Die Vermieterin vermietete der Mieterin ab dem 24. Juli 1933 für ein Jahr diverse Räumlichkeiten für Lagerung, Büro usw. zu einem jährlichen Mietzins von Fr. 13'000. Es wurde weiter vereinbart, dass die Räumlichkeiten an ein Lebensmittelgeschäft untervermietet werden dürften und sich der Mietvertrag jeweils durch stillschweigende Vereinbarung von Jahr zu Jahr verlängern würde. Die Migros S. A. übernahm am 4. August 1933 den Mietvertrag und begann, die Räumlichkeiten einzurichten. Am 10. November 1933 verbot der Grosse Rat des Kantons Waadt

20 BGE 57 II 532 Sachverhalt A S. $532 \mathrm{ff}$.

21 BGE 57 II 532 E. 1 S. $534 \mathrm{ff}$. 
die Eröffnung von Filialen der Migros S. A. auf Waadtländer Territorium. Die Migros S. A. erhielt in der Folge keine Genehmigung für den Betrieb ihres Geschäfts. Nach Bezahlung zweier Quartalsmieten teilte sie dem Vermieter am 20. Januar 1934 mit, dass es ihr unmöglich sei, die Räumlichkeiten zweckgemäss zu nutzen und folglich die Miete zu zahlen. Die Vermieterin war damit nicht einverstanden und forderte die Bezahlung des gesamten Jahreszinses. Erstinstanzlich wurde die Migros S. A. verpflichtet, den vollen Mietzins zu bezahlen. Das Bundesgericht wies die gegen diesen Entscheid gerichtete Beschwerde der Migros S. A. ab. ${ }^{22}$

[27] Es nahm in seinen Erwägungen auf den BGE 57 II 532 Bezug und legte dar, weswegen im Gegensatz zu jenem Entscheid im zu beurteilenden Fall Art. 119 Abs. 2 OR nicht anzuwenden sei und der Mietzins vollumfänglich geschuldet bleibe: «Il est vrai que, dans cette cause [BGE 57 II 532], le Tribunal fédéral a appliqué l'art. 119 à l'obligation du preneur de payer le loyer, mais il y a entre les deux espèces des différences essentielles. Par le contrat passé entre le propriétaire Emrich et Ia dentiste non diplômée Dame Schmitzberger, celle-ci non seulement louait du premier un chalet à Mollis près de Weesen pour y ouvrir un cabinet dentaire, elle reprenait encore Ia clientèle de son bailleur, (die bisher geführte Praxis), au prix global de 500 francs par mois. L'exploitation des locaux loués pour l'exercice de l'art dentaire était une clause essentielle (eine Voraussetzung) du contrat [...] et la possibilité de faire de Ia chose louée l'usage stipulé par la locataire était promise par Ie bailleur, c'était une assurance donnée (eine Zusicherung). Par suite d'un changement de la législation cantonale, ces prévisions ne se sont pas réalisées à partir d'un moment donné: pour un motif dont le preneur et cessionnaire n'avait pas a répondre, l'utilisation du chalet aux fins spécifiées et partant Ia jouissance de la clientère cédée sont devenues impossibles, et l'art. 119 al. 2 a été appliqué. Dans le cas de la Migros rien de pareil n'a été stipulé ni promis: il s'agit d'un bail pur et simple de Iocaux, sans précision d'un usage spécial; on s'est borné à prévoir la sous-Iocation à une maison d'alimentation.» ${ }^{23}$

\subsubsection{Schlussfolgerung}

[28] Im älteren Fall anerkannte das Bundesgericht die vorzeitige Beendigung des Mietvertrags infolge Zweckverfehlung, während es im einige Jahre später ergangenen Entscheid von blosser Verwendungsunmöglichkeit ausging und dementsprechend befand, die Mietzinse seien trotz untersagter Geschäftstätigkeit vollumfänglich geschuldet. Für das Bundesgericht waren diese beiden Fälle deswegen unterschiedlich zu beurteilen, weil im älteren Entscheid die Mieterin die bisher geführte Praxis mitsamt Kundenstamm übernommen hatte, der Zweck des Betreibens der Zahnarztpraxis eine wesentliche Voraussetzung des Vertragsschlusses gewesen war und vor allem der Vermieter die Möglichkeit des Betriebs dieser Praxis zugesichert hatte. Im später ergangenen Entscheid handelte es sich dagegen für das Bundesgericht um eine gewöhnliche Raummiete, da die Verwendungsmöglichkeit im Vertrag nicht spezifisch geregelt wurde.

[29] Wird diese Rechtsprechung auf die im Zusammenhang mit dem Coronavirus aufgekommenen Fälle angewendet, ergibt sich folgende Rechtslage:

[30] Um im Einzelfall konkret beurteilen zu können, ob die Leistung des Vermieters infolge der staatlich angeordneten Betriebsschliessungen unmöglich geworden ist, muss der Inhalt der von

22 BGE 62 II 42 Sachverhalt A S. $42 \mathrm{ff}$.

23 BGE 62 II 42 E. 1 S. 44 ff. 
ihm geschuldeten Leistungspflicht festgestellt werden. ${ }^{24}$ Mittels Vertragsauslegung ist das genaue Leistungspflichtenprogramm zu ermitteln.

[31] Selten werden Mietverträge über Geschäftsräumlichkeiten abgeschlossen, ohne dass zugleich ein spezifischer Verwendungszweck vereinbart wird. Ist dies jedoch ausnahmsweise der Fall, so ist die Rechtslage klar. Analog wie in BGE 62 II 42 hat diesfalls der Mieter das Verwendungsrisiko zu tragen. Der Mietzins bleibt vollumfänglich geschuldet.

[32] Wenn die Mietparteien - was der Regelfall ist - miteinander spezifisch vereinbaren, wozu die Gewerberäume gemietet werden, ist die Rechtslage etwas unklarer. Indem die Parteien eine bestimmte Verwendung miteinander beschliessen, legen sie nämlich gemeinsam fest, wofür der Mieter die ihm zum Gebrauch überlassene Sache verwenden darf. Wird z.B. die Verwendung der Räumlichkeiten für den Betrieb eines Kosmetikstudios ( $D a s$ Mietobjekt steht dem Mieter für das Führen eines Ladengeschäftes mit Studio für kosmetische Behandlungen zur Verfügung.») vereinbart, und diese infolge der staatlich angeordneten Betriebsschliessungen temporär verboten, so stellt sich die Frage, ob von unverschuldeter nachträglicher Unmöglichkeit der vom Vermieter geschuldeten Leistung auszugehen ist. Mit Blick auf die aufgezeigte Rechtsprechung der Zwischenkriegszeit ist dies jedoch nicht der Fall. Es ist von blosser Verwendungsunmöglichkeit - und nicht von Zweckverfehlung - auszugehen. Wenn der Staat die spezifisch vereinbarte Verwendung temporär untersagt, so begeht der Vermieter grundsätzlich keine Pflichtverletzung. ${ }^{25}$ Seine Leistung bleibt möglich bzw. wird weiterhin erbracht (Überlassen vertragskonformer Geschäftsräume).

[33] Die Rechtslage ist erst dann anders zu beurteilen, wenn der Vermieter über die herkömmliche Gebrauchsüberlassungspflicht hinausgehende vertragliche Zusicherungen gemacht (z.B. Garantie, dass der Mieter die vorgesehene Geschäftstätigkeit auch tatsächlich ausüben kann) oder entsprechende vertragliche Risikotragungsklauseln mit dem Mieter getroffen hat (vgl. hierzu Titel 3.1).

[34] Somit dürfte es sich für die meisten Geschäftsmieter nicht lohnen, sich auf Unmöglichkeit zu berufen. Erstens ist fraglich, ob Art. 119 OR überhaupt auf vorübergehende Unmöglichkeiten anwendbar ist. Zweitens gehört die tatsächliche Verwendungsmöglichkeit nur dann zur Leistungspflicht des Vermieters, wenn er sie dem Mieter zugesichert hat. Das dürfte nur selten der Fall sein.

\subsection{Möglichkeit 2: Herabsetzungsanspruch wegen Mangel an der Mietsache}

[35] Ergeht ein öffentlich-rechtliches Betriebsverbot, stellt sich die Frage, ob nach Massgabe der allgemeinen Regeln vertragliche Herabsetzungsansprüche geltend gemacht werden können (Art. 259d OR). Schadenersatzansprüche fallen mangels vorwerfbaren Verhaltens der Parteien von Vornherein ausser Betracht.

[36] Die in Art. 259d OR geregelte Mietzinsherabsetzung verfolgt den Zweck, dem durch Mängel an der Mietsache verminderten Gebrauchswert mittels einer Anpassung des Mietzinses Rechnung zu tragen und damit das Gleichgewicht zwischen den vereinbarten Leistungen wiederherzustellen. Der Herabsetzungsanspruch setzt kein Verschulden des Vermieters hinsichtlich des

24 Vgl. Koller (Fn. 17), N 53.09.

25 A.M. Brutschin/Rubli/Stastny (Fn. 6), N 2.9. 
Mangels an der Mietsache voraus. ${ }^{26}$ Mängel, die vom Mieter zu vertreten oder von ihm zu beseitigen sind, fallen ausser Betracht. Der Vermieter muss zudem Kenntnis vom Mangel haben, was im Zusammenhang mit den behördlich angeordneten Betriebsschliessungen stets der Fall sein dürfte.

\subsubsection{Abgrenzung und Verhältnis zu Art. 119 OR}

[37] In der Lehre wird teilweise die Ansicht vertreten, der Herabsetzungsanspruch sei eine Konkretisierung des Grundgedankens von Art. 119 Abs. 2 OR. ${ }^{27}$ Für Higi/WiLdisen ist diese Auffassung insofern sachgerecht, als unter «Grundgedanke von Art. 119 Abs. 2» lediglich die Ausgleichsregelung von Leistungsstörungen bei zweiseitigen Verträgen verstanden wird. Art. 119 OR befasse sich konkret mit der (objektiven) Unmöglichkeit. Diese sei hingegen nicht Voraussetzung des Herabsetzungsanspruchs. ${ }^{28}$ Daraus und auch unter Berücksichtigung des Grundsatzes des Vorrangs der speziellen Norm lässt sich schliessen, dass der Herabsetzungsanspruch gemäss Art. 259d OR in den hier interessierenden Fällen grundsätzlich eher zur Anwendung kommt als Art. 119 OR.

\subsubsection{Begriff des Mangels}

[38] Der Mangel am Mietobjekt ist im Gesetz nicht näher definiert. Nach bundesgerichtlicher Rechtsprechung weist ein Mietobjekt einen Mangel auf, «wenn ihm eine vertraglich zugesicherte oder sich aus dem vertraglichen Gebrauchszweck ergebende Eigenschaft fehlt. Worin der vorausgesetzte Gebrauch besteht und welchen Zustand der Mietsache die Mieter erwarten dürfen, ergibt sich primär aus der Parteivereinbarung [... ]. Auch Mängel, die nicht in der Mietsache selbst begründet sind, sondern sich aus der Umwelt oder aus dem Verhalten Dritter ergeben, können einen Mangel der Mietsache darstellen». ${ }^{29}$ Art. 259a Abs. 1 OR stellt dem Mangel die Störung im vertragsgemässen Gebrauch gleich. Damit wollte der Gesetzgeber zum Ausdruck bringen, dass neben dem eigentlichen körperlichen Sachmangel auch jede andere Störung im vertragsgemässen Gebrauch den Mangeltatbestand erfüllen kann. Unter anderem kommen auch Störungen rechtlicher Natur in Betracht. ${ }^{30}$

[39] Kernfrage ist daher, ob die staatlich angeordneten Betriebsschliessungen als Mangel am Mietobjekt oder Störung im vertragsgemässen Gebrauch desselben zu betrachten sind. Mit der Beantwortung dieser Frage steht und fällt grundsätzlich der Herabsetzungsanspruch.

[40] Aufschlussreich ist in diesem Zusammenhang die Rechtsprechung des deutschen Bundesgerichtshofs («BGH»). Dieser unterscheidet zwischen objekt- und betriebsbezogenen Umständen. Die Verantwortung des Vermieters beschränkt sich auf die objektbezogenen Umstände, nament-

26 ZK OR-Higi/Wildisen (Fn. 12), Art. 259d N 5.

27 Alfred Koller, in: Theo Guhl, Das Schweizerische Obligationenrecht, 9. Aufl., Zürich 2000 (zit. Guhl/Bearbeiter), S. $415 \mathrm{~N} 53$.

28 ZK OR-Higi/WLdisen (Fn. 12), Art. 259d N 5.

29 Urteil des Bundesgerichts 4C.39/2003 vom 23. April 2003 E. 4.

30 Hans Giger, in: Berner Kommentar zum schweizerischen Privatrecht, Obligationenrecht, Die Miete, Art. 253-273c OR, Art. 256-259i OR, Bern 2015 (zit. BK-GIger), Normspezifische Vorbemerkungen zu Art. 258-259i N 38. 
lich solche, die sich auf die Lage, Beschaffenheit oder den Zustand der Mietsache beziehen. ${ }^{31}$ In einem Entscheid aus dem Jahre 2011 zur Auswirkung des Inkrafttretens der Nichtraucherschutzgesetze auf einen laufenden Pachtvertrag über eine Gaststätte legte der BGH diese Rechtsprechung dar. ${ }^{32}$ Die Gaststättenbetreiber befürchteten infolge der in den Ländern eingeführten Regelungen zum Nichtraucherschutz in öffentlichen Gebäuden ein Ausbleiben der Gäste und einen damit zusammenhängenden erheblichen Umsatzrückgang. Es entbrannte eine Diskussion, ob die mit den Nichtraucherschutzgesetzen der Länder verbundene Beschränkung einen Sachmangel der verpachteten Gaststätten begründe und dementsprechend die Pächter zur Minderung der Pacht berechtige. Der BGH folgte dieser Auffassung nicht. In seinem Entscheid führte er aus:

«Ergeben sich aufgrund von gesetzgeberischen Maßnahmen während eines laufenden Pachtverhältnisses Beeinträchtigungen des vertragsmäßigen Gebrauchs eines gewerblichen Pachtobjekts, kann dies nachträglich einen Mangel iSv §§ 581 Abs.2, 536 Abs.1 Satz 1 BGB begründen [...]. Voraussetzung hierfür ist jedoch, dass die durch die gesetzgeberische Maßnahme bewirkte Gebrauchsbeschränkung unmittelbar mit der konkreten Beschaffenheit, dem Zustand oder der Lage des Pachtobjekts in Zusammenhang steht. Andere gesetzgeberische Maßnahmen, die den geschäftlichen Erfolg beeinträchtigen, fallen dagegen in den Risikobereich des Pächters [... ]. Denn der Verpächter von Gewerberäumen ist gemäß \$§581 Abs. 2, 535 Abs. 1 Satz 2 BGB lediglich verpflichtet, den Pachtgegenstand während der Vertragslaufzeit in einem Zustand zu erhalten, der dem Pächter die vertraglich vorgesehene Nutzung ermöglicht. Das Verwendungsrisiko bezüglich der Pachtsache trägt bei der Gewerberaummiete dagegen grundsätzlich der Mieter. [... ]. Dazu gehört vor allem das Risiko, mit dem Pachtobjekt Gewinne erzielen zu können. Erfüllt sich die Gewinnerwartung des Pächters aufgrund eines nachträglich eintretenden Umstandes nicht, so verwirklicht sich damit ein typisches Risiko des gewerblichen Pächters. Das gilt auch in Fällen, in denen es durch nachträgliche gesetzgeberische oder behördliche Maßnahmen zu einer Beeinträchtigung des Gewerbebetriebs des Pächters kommt.»33

[41] Das gesetzliche Rauchverbot führte demnach nicht zu einem Mangel an der verpachteten Gaststätte. ${ }^{34}$ Die mit dem gesetzlichen Rauchverbot zusammenhängende Gebrauchsbeschränkung beruhte nicht auf der konkreten Beschaffenheit der Pachtsache, sondern betraf vielmehr die betrieblichen Verhältnisse des Pächters. Die massgeblichen Regelungen knüpften allein an die Art der Nutzung der Gebäude oder Gebäudeteile und den Umstand, dass dort Publikumsverkehr herrschte, an. ${ }^{35}$ Die baulichen Gegebenheiten der betroffenen Gebäude oder Gebäudeteile waren für die Geltung des Rauchverbots unerheblich. Dieses bezog sich nur auf die Art und Weise der Betriebsführung des Pächters und stellte damit keine objektsbezogene Gebrauchsbeschränkung dar, für die der Verpächter verantwortlich gewesen wäre. ${ }^{36}$

31 Peter GüNTER, Der Einfluss öffentlich-rechtlicher Verpflichtungen auf mietvertragliche Vereinbarungen, Risikoverteilung, Gestaltungsmöglichkeiten der Mietvertragsparteien und Rechtsstellung des Mieters bei Nichterfüllung von Vermieterpflichten, NZM 2016, S. 569 ff., S. 571. 
[42] Ähnlich argumentiert ein Teil der Schweizer Lehre. So schreibt HigI, das vom Mieter betriebene Geschäft sei nicht Bestandteil der mietvertraglichen Abmachungen, sondern es sei unabhängig von diesem als Teil der Rechtssphäre des Mieters zu betrachten. ${ }^{37}$ Werde nun dem Mieter kraft behördlicher Anordnung der Betrieb seines Geschäfts untersagt, so sei der Grund hierfür in der ausgeübten Geschäftstätigkeit zu suchen, und nicht im Zustand des Mietlokals. In ähnlicher Weise befinden Haefeli/Galli/Vischer, dass beim Veranstaltungsverbot und bei der Schliessungsanordnung kein Schlechterfüllungstatbestand vorliege, weil es bei diesen um betriebsbezogene Eigenschaften gehe. ${ }^{38}$

\subsubsection{Schlussfolgerung}

[43] Die Rechtsprechung des BGH zur Unterscheidung von betriebs- und objektbezogenen Umständen überzeugt. Bei objektbezogenen Umständen anerkennt auch die schweizerische Rechtsprechung Mängel und gewährt Herabsetzungsansprüche. Entsprechende Senkungsbegehren wurden schon bei Lärm durch Bauvorhaben in der Nachbarschaft ${ }^{39}$ oder etwa bei Beeinträchtigungen durch Fluglärm von den Gerichten anerkannt. ${ }^{40}$ Staatlich angeordnete Betriebsschliessungen im Zusammenhang mit der Bekämpfung des Coronavirus sind jedoch betriebsbezogen. Bei diesen geht es nicht um die Lage, den Zustand oder die Beschaffenheit des Mietobjekts, sondern um das Geschäft des Mieters.

[44] Hinzuweisen ist noch darauf, dass bei Fehlen einer zugesicherten Eigenschaft von einem Mangel auszugehen ist. ${ }^{41}$ Zugesicherte Eigenschaften des Objekts bilden nämlich Teil der vertraglich festgelegten Leistungspflicht des Vermieters und sind daher bei der Bestimmung des vertragsgemässen Zustands der Mietsache zu berücksichtigen. ${ }^{42} \mathrm{Ob}$ eine Eigenschaft zugesichert worden ist, bestimmt sich nach der vertraglichen Vereinbarung. Eine mögliche Zusicherung, die im Zusammenhang mit staatlich angeordneten Betriebsschliessungen von Bedeutung sein kann, ist z.B. diejenige, dass sich ein bestimmter Umsatz erzielen lasse, ${ }^{43}$ oder dass das Risiko einer Betriebsschliessung vom Vermieter getragen werde.

\subsection{Möglichkeit 3: Vertragsanpassung, clausula rebus sic stantibus}

[45] Ist es dem Mieter wegen der behördlich angeordneten Betriebsschliessung nicht möglich, seine Geschäftstätigkeit auszuüben, kann er gegebenenfalls eine Vertragsanpassung aufgrund geänderter Umstände geltend machen. Der Vertrag wird jedoch nur unter der Voraussetzung angepasst, dass die Erfüllung desselben für den Mieter nicht mehr zumutbar ist, insbesondere weil eine gravierende Störung des Verhältnisses von Leistung und Gegenleistung (Äquivalenzstörung)

\footnotetext{
Higi (Fn. 8), S. 1.

Haefeli/Galli/Vischer (Fn. 14), N 29 und 30.

Mietrechtspraxis/mp 3/87 S. 51.

Vgl. Mietrechtspraxis/mp 1/02 S. 29 ff., Mietgericht Bezirk Bülach vom 24. September 2001.

ZK OR-Higi/WiLdisen (Fn. 12), Art. 258 N 29.

ZK OR-Higi/WiLdisen (Fn. 12), Art. 258 N 29.

Vgl. hierzu den Nachweis bei ZK OR-Higi/Wildisen (Fn. 12), Art. 259d N 49.
} 
besteht. Das Institut der Vertragsanpassung wird in der Schweiz seit langer Zeit anerkannt und aus Art. 2 ZGB hergleitet. ${ }^{44}$

\subsubsection{Abgrenzung und Verhältnis zu weiteren Rechtsinstituten}

[46] Beim Vertragsschluss gehen die Parteien von bestimmten Umständen und Annahmen aus. Erweisen sich diese in einem späteren Zeitpunkt als unzutreffend, so stellt sich die Frage, ob der Vertrag wie ursprünglich vorgesehen abgewickelt werden soll und kann. Grundsätzlich sind Verträge zu halten, wie sie geschlossen wurden («pacta sunt servanda»). Nur in wenigen Ausnahmefällen kann von diesem Grundsatz abgewichen werden. ${ }^{45}$ Dabei steht der Grundgedanke im Vordergrund, dass die Parteien den Vertrag nicht so wie erfolgt abgeschlossen hätten, wenn sie nicht falschen Vorstellungen über die Zustände bei Vertragsschluss oder über die Entwicklung der Verhältnisse unterlegen wären. Folgende Rechtsfiguren des schweizerischen Rechts basieren auf diesem Gedanken:

- der Grundlagenirrtum gemäss Art. 24 Abs. 1 Ziff. 4 OR;

- die Kündigung aus wichtigem Grund gemäss Art. 266g OR;

- die Vertragsanpassung wegen veränderter Verhältnisse (clausula rebus sic stantibus).

[47] Der Grundlagenirrtum kommt im vorliegenden Zusammenhang nicht in Betracht, weil es an einer konkreten Fehlprognose über einen hinreichend bestimmten Sachverhalt mangelt. ${ }^{46}$ Die betroffenen Vertragsparteien haben sich im Zeitpunkt des Vertragsschlusses kaum konkrete Vorstellungen über das Eintreten oder Nichteintreten von Pandemien gemacht.

[48] Das Vorgehen über die Kündigung aus wichtigem Grund gemäss Art. 266g OR dürfte kaum zielführend sein. Das Interesse der Mieter ist regelmässig darauf gerichtet, den Mietvertrag über die Coronavirus-Krise hinaus weiterzuführen. Darüber hinaus ist fraglich, ob und falls ja, unter welchen Umständen überhaupt von einem wichtigen Grund auszugehen ist. Die Kündigung aus wichtigem Grund kommt nur dann in Betracht, wenn die Unzumutbarkeit einen anderen Grund hat als die Äquivalenzstörung (zwischen Leistung und Gegenleistung), da es sonst zu einem Wertungs- und Voraussetzungskonflikt mit der clausula rebus sic stantibus kommt. ${ }^{47}$ Die Covid-19-bedingten Betriebsschliessungen betreffen die Thematik der Leistungsäquivalenz. Deshalb ist unseres Erachtens die Kündigung aus wichtigem Grund nicht anwendbar.

44 Wolfgang Wiegand, in: Basler Kommentar Obligationenrecht I, 7. Aufl., Basel 2019 (zit. BSK OR I-Verfasser), Art. 18 N 95. S. zur clausula rebus sic stantibus als Instrument der Vertragsanpassung auch die Hinweise bei Bernhard Stehle, in: Gauch/Aepli/Stöckli, Präjudizienbuch OR, 9. Aufl., Zürich 2016, Art. 18 N 65 ff. BSK OR I-WIEgAND (Fn. 44), Art. 18 N 97.

46 Urteil des Bundesgerichts 4C.34/2000 vom 24. April 2001 E. 3c/bb (in BGE 127 III 300 nicht publ. Erw.): der Irrende muss «sich über einen bestimmten Sachverhalt geirrt, d.h. eine konkrete Fehlprognose gestellt» haben. Grundlegend BGE 109 II 105; s. sodann BGE 118 II 297.

47 S. BGE 128 III 428 E. 3c S. 432: «Die clausula setzt Veränderungen der äusseren Umstände voraus, von denen alle Vertragsparteien gleichermassen betroffen sind, und die zu einer gravierenden Äquivalenzstörung geführt haben. Im Gegensatz dazu hat die Kündigung aus wichtigem Grund keine Äquivalenzstörung zur Voraussetzung. Im Vordergrund steht vielmehr die Frage, ob das Gebundensein an den Vertrag für die Partei wegen veränderter Umstände ganz allgemein unzumutbar geworden ist, also nicht nur unter wirtschaftlichen, sondern auch unter anderen die Persönlichkeit berührenden Gesichtspunkten.» 
[49] Im Vordergrund steht demzufolge die Vertragsanpassung. Erlaubt sei an dieser Stelle der Hinweis, dass, wie bereits vorne unter Titel 3.3 erwähnt, Art. 119 OR nur auf Fälle dauernder Unmöglichkeit anwendbar ist. ${ }^{48} \mathrm{Im}$ Gegensatz dazu können Verträge grundsätzlich auch bei nur vorübergehender Änderung der Umstände angepasst werden.

\subsubsection{Voraussetzungen der Anpassung}

[50] Gemäss Rechtsprechung setzt «[e] in richterlicher Eingriff in einen Vertrag aufgrund veränderter Umstände [...] voraus, dass die Verhältnisänderung weder vorhersehbar noch vermeidbar war, [...] eine gravierende Äquivalenzstörung zur Folge hat und der Vertrag nicht vorbehaltlos erfüllt wurde». ${ }^{49}$

[51] Veränderte Umstände: Demgemäss ist zunächst zu fragen, ob sich die Verhältnisse seit Vertragsschluss geändert haben. Diese Frage ist in den hier interessierenden Fällen zweifellos zu bejahen. Aufgrund der weltweiten Pandemie und der infolgedessen von staatlicher Seite angeordneten Betriebsschliessungen haben sich für zahlreiche betroffene Geschäftsraummieter die Umstände seit Vertragsschluss erheblich geändert. Sie dürfen die Räumlichkeiten nicht mehr wie gewohnt nutzen, z.B. nicht mehr als Coiffeursalon oder Restaurantbetrieb.

[52] Voraussehbarkeit: In einem nächsten Schritt ist zu prüfen, ob diese Verhältnisänderung vorausgesehen werden konnte. Bei langfristigen Verträgen müssen «die Parteien [nämlich] damit rechnen, dass sich die zur Zeit des Vertragsabschlusses bestehenden Verhältnisse später ändern. Namentlich Änderungen der Gesetzeslage gelten grundsätzlich nicht als unvorhersehbar [...]. Sehen die Parteien ausdrücklich oder sinngemäss davon ab, den Einfluss solcher Änderungen auf die gegenseitigen Leistungen auszuschliessen, so entspricht es grundsätzlich dem Wesen des Vertrages, dass er so erfüllt wird, wie er abgeschlossen worden ist».$^{50}$ Grundsätzlich ist mit dem Ausbruch von Epidemien resp. Pandemien und dadurch bedingten Betriebsschliessungen jederzeit zu rechnen. In Versicherungspolicen sind entsprechende Risiken regelmässig geregelt, oftmals gar detaillierte Deckungsausschlüsse dazu formuliert.

[53] Gemäss Rechtsprechung ist «die Voraussehbarkeit [jedoch] zu verneinen, wenn eine Verhältnisänderung [...] als solche zwar vorhersehbar war, nicht aber deren Art, Umfang und Auswirkungen auf den Vertrag». ${ }^{51}$ Noch vor kurzer Zeit rechnete kaum jemand in der Schweiz beim Abschluss eines Mietvertrags mit staatlich angeordneten Betriebsschliessungen aufgrund einer grassierenden Pandemie, die für längere Zeit andauern. Die Parteien haben solche Entwicklungen im Zeitpunkt des Vertragsschlusses im Regelfall nicht in Betracht gezogen und mussten dies auch nicht.

[54] Aufschlussreich ist ein Entscheid des Bundesgerichts aus dem Jahre 1922.52 Das Bundesgericht hielt die wirtschaftlichen Umwälzungen nach dem ersten Weltkrieg für nicht voraussehbar und gewährte infolgedessen dem Pächter eine erhebliche Anpassung des geschuldeten Zinses unter Anwendung der clausula rebus sic stantibus. Im Wesentlichen begründete es seinen Entscheid folgendermassen:

\footnotetext{
48 Urteil des Bundesgerichts 4C.34/2000 vom 24. April 2001 E. 4 (in BGE 127 III 300 nicht publ. Erw.).

49 BGE 127 III 300 E. 5b S. 304.

50 BGE 127 III 300 E. 5b/aa) S. 305.

51 BGE 127 III 300 E. 5b/aa) S. 305

52 BGE 48 II 252.
} 
"Nach den vom Bundesgericht in zahlreichen Entscheiden ausgesprochenen Grundsätzen kann eine Leistungserschwerung dann zur gänzlichen oder teilweisen Befreiung des Schuldners führen, wenn sich die Verhältnisse derart geändert haben, dass es sich für ihn um eine vom wirtschaftlichen Gesichtspunkte aus ganz andere, beziehungsweise um eine Leistung handelt, die ihm nach Treu und Glauben im Verkehr nicht mehr zugemutet werden darf. Diese Voraussetzungen hat die Vorinstanz hier mit Recht bejaht. Dem Expertengutachten ist zu entnehmen, dass die Personenfrequenz in den Jahren 1914-1917 gegenüber 1910-13 um 43,93\% zurückging und die Fahrleistungen der Dampfschiffe um 31,48\% vermindert wurden, woraus sich für den Beklagten ein Einnahmenausfall von 52,33\% ergab. Diese infolge des Krieges eingetretene ausserordentliche wirtschaftliche Umwälzung war für die Parteien beim Vertragsabschluss nicht voraussehbar; es muss daher auch als ausgeschlossen gelten, dass sich ihr Wille auf die Wirkungen dieser ausserhalb jeder menschlichen Erkenntnis liegenden Ereignisse bezogen hätte, sodass von einer Übernahme der Gefahr schlechthin durch den Beklagten, für die übrigens auch der Vertrag keine Anhaltspunkte bietet, jedenfalls keine Rede sein kann.» ${ }^{53}$

[55] Vermeidbarkeit: Rechnen die Parteien bei Vertragsabschluss mit künftigen Ereignissen, können sie für diesen Fall eine Anpassung vertraglich vorsehen. ${ }^{54}$ Unter den Begriff der Vermeidbarkeit fällt auch die Überwindbarkeit: Hätte eine Partei die Folgen der künftigen Entwicklung überwinden können, so fällt die Vertragsanpassung ausser Betracht. ${ }^{55}$ Allgemein wurde nicht mit einem Ereignis wie der Coronavirus-Pandemie gerechnet. Auch ist grundsätzlich nicht ersichtlich, wie die Mieter die Folgen des Lockdowns überwinden könnten. Immerhin ist zuzugeben, dass die Mieter gewisse Tätigkeiten noch ausüben können. So kann ein Restaurant die Räumlichkeiten zumindest teilweise noch als Take-away oder Hauslieferdienst nutzen. Um die Vermeidbarkeit zu bejahen, reicht dies jedoch nicht aus. Vielmehr muss diesen Umständen bei der Festsetzung des Ausmasses der Anpassung Rechnung getragen werden (Schadenminderungspflicht).

[56] Gravierende Äquivalenzstörung: Durch die Veränderung der Verhältnisse muss eine schwere Störung des Vertragsverhältnisses ausgelöst werden. In Mietverhältnissen geht es um den Austausch von vermögenswerten Leistungen. Es muss in solchen Fällen ein grobes Missverhältnis zwischen Leistung und Gegenleistung eintreten. ${ }^{56}$ Diese Voraussetzung dürfte in vielen der vorliegend interessierenden Fälle gegeben sein. Die volle Bezahlung des Mietzinses kann unverhältnismässig werden, wenn die Räumlichkeiten infolge staatlicher Anordnung nicht oder praktisch nicht mehr genutzt werden können und der Mieter mit dem Mietobjekt entsprechend keine (oder nur noch weit geringere) Einnahmen mehr erzielen kann. Es wird dadurch für den Mieter schwieriger, seine eigene Leistung zu erbringen. Diese Schwierigkeiten erreichen in vielen Fällen die Schwelle der Unzumutbarkeit.

53 BGE 48 II 252 E. 1 S. 252.

54 BGE 135 III 1 E. 2.5 S. 10.

55 S. Art. 79 Abs. 1 des Wiener Kaufrechts: «Eine Partei hat für die Nichterfüllung einer ihrer Pflichten nicht einzustehen, wenn sie beweist, dass die Nichterfüllung auf einem ausserhalb ihres Einflussbereichs liegenden Hinderungsgrund beruht und dass von ihr vernünftigerweise nicht erwartet werden konnte, den Hinderungsgrund bei Vertragsabschluss in Betracht zu ziehen oder den Hinderungsgrund oder seine Folgen zu vermeiden oder zu überwinden.» 
[57] Keine vorbehaltslose Erfüllung des Vertrags: Wenn betroffene Mieter gestützt auf die clausula rebus sic stantibus die Anpassung des Mietzinses verlangen möchten, dürfen sie die Miete während des Andauerns der staatlich angeordneten Betriebsschliessungen nur unter Vorbehalt der Vertragsanpassung bezahlen.

[58] Insgesamt scheinen die Voraussetzungen der clausula rebus sic stantibus und damit der Vertragsanpassung in zahlreichen Fällen im Zusammenhang mit Coronavirus-bedingten Betriebsschliessungen gegeben zu sein. Infolgedessen rückt die Frage nach dem sachgerechten Ausmass der Anpassung in den Vordergrund.

\subsubsection{Ausmass der Anpassung}

[59] Das Gericht hat zu ermitteln, was die Parteien nach dem Grundsatz von Treu und Glauben vereinbart hätten, wenn sie den eingetretenen Verlauf der Dinge in Betracht gezogen hätten. «Dabei hat es sich am Denken und Handeln vernünftiger und redlicher Vertragspartner sowie an Wesen und Zweck des konkret in Frage stehenden Vertrages zu orientieren.» ${ }^{57}$

[60] $\mathrm{Zu}$ würdigen sind die Umstände im Einzelfall. Es ist zu berücksichtigen, inwiefern der Mieter gewisse Räumlichkeiten noch vertragsgemäss nutzen kann. Die Lagerung von Produkten ist grundsätzlich auch während des Lockdowns möglich. Dafür ist ein Anteil des Mietzinses zu bezahlen. In vielen Fällen dürften auch Büroarbeiten - wenn auch gegebenenfalls nur in eingeschränktem Masse - noch möglich sein. Zudem stellt sich die Frage nach der Schadenminderungspflicht. Zumutbare Massnahmen sind bei der Vertragsanpassung zu berücksichtigen (da sich das Gericht am Denken und Handeln vernünftiger und redlicher Vertragspartner orientiert). Bei Restaurants ist z.B. zu prüfen, ob der Mieter nicht zumindest die Küche für die Zubereitung von Mahlzeiten hätte nutzen können, um als Take-away-Betrieb oder Hauslieferdienst tätig zu sein (dabei spielen Kosten-Nutzen-Erwägungen eine wichtige Rolle). Wird die Zeit des Lockdowns genutzt, um grössere Umbauprojekte anzugehen, ist zu berücksichtigen, inwiefern auch bei normaler Nutzung der Betrieb eingeschränkt gewesen wäre.

\section{Fazit}

[61] Die Rechtsfigur der nachträglichen unverschuldeten Unmöglichkeit ist auf die hier interessierenden Fälle nicht anwendbar, insbesondere weil es nicht um eine dauerhafte Unmöglichkeit geht. Die staatlich angeordneten Betriebsschliessungen sind nur vorübergehender Natur. Zudem verunmöglichen die Betriebsschliessungen die Leistung des Vermieters in der Regel nicht, weil die tatsächliche Verwendungsmöglichkeit nur dann zur Leistungspflicht des Vermieters gehört, wenn er sie dem Mieter zugesichert hat.

[62] Auch der Herabsetzungsanspruch wegen Mängeln an der Mietsache gemäss Art. 259d OR hat nur in denjenigen Fällen eine Bedeutung, in welchen der Vermieter im Zusammenhang mit Pandemien spezifische Zusicherungen abgegeben oder zu seinen Ungunsten vertragliche Vereinbarungen mit dem Mieter getroffen hat.

57 BGE 127 III 300 E. 6a S. 307; ebenso BGE 143 III 558 E. 4.1.1 S. 562; 115 II 484 E. 4b S. 488. 
[63] Die grösste Bedeutung kommt wohl der Vertragsanpassung gemäss den Voraussetzungen der clausula rebus sic stantibus zu. Die im Zusammenhang mit der Bekämpfung der Ausbreitung des Coronavirus angeordneten Betriebsschliessungen waren in der Regel weder voraussehbar noch vermeidbar. Es resultierten in zahlreichen Mietverhältnissen erhebliche Missverhältnisse zwischen Leistung und Gegenleistung. Es rechtfertigt sich, die entsprechenden Verträge anzupassen. [64] Bei der Anpassung sind die Umstände des konkreten Einzelfalls massgebend. Mieter müssen sich anrechnen lassen, was sie noch nutzen können. Insbesondere Lagerflächen, Büroräumlichkeiten usw. sind auch während den staatlich angeordneten Betriebsschliessungen benutzbar. Darüber hinaus gilt die Schadenminderungspflicht. Mieter müssen soweit möglich und zumutbar Einnahmenausfälle aufgrund des Lockouts kompensieren.

[65] Es ist schwierig abzuschätzen, wie Gerichte dereinst die Coronavirus-Mietstreitigkeiten entscheiden werden. Mit der Vertragsanpassung gestützt auf die Voraussetzungen der clausula rebus sic stantibus steht den Rechtsanwendern ein Instrument zur Verfügung, mit welchem im Einzelfall sachgerechte und pragmatische Lösungen möglich sind.

Dr. iur. Sebastian Reichle, Rechtsanwalt, ist Partner bei Reichle Stehle Rechtsanwälte, St. Gallen, Lehrbeauftragter an der Universität St. Gallen und der Fachhochschule St. Gallen sowie Geschäftsführer am Institut für Rechtswissenschaft und Rechtspraxis (IRP-HSG).

Dr. iur. Bernhard Stehle, Rechtsanwalt, ist Partner bei Reichle Stehle Rechtsanwälte, St. Gallen, und Lehrbeauftragter an der Universität St. Gallen. 